\title{
Management of refractory or relapsed primary central nervous system lymphoma (Review)
}

\author{
RYUYA YAMANAKA \\ Research Center of Innovative Cancer Therapy, Kurume University School of Medicine, \\ Kurume, Fukuoka 830-0011, Japan
}

Received May 7, 2009; Accepted August 14, 2009

DOI: 10.3892/mmr_00000186

\begin{abstract}
Primary central nervous system lymphoma(PCNSL) is a non-Hodgkin's lymphoma arising in the central nervous system. Combined methotrexate-based chemoradiotherapy is the standard treatment for PCNSL, resulting in a median overall survival of 25-51 months. Failure of first-line treatment has been reported in most patients with PCNSL. Salvage therapy improves outcome; however, since many different treatments have been applied, conclusions cannot be drawn regarding an optimal treatment schedule. This review analyzes the efficacy of different salvage therapies that have been reported in the literature. These well-designed, randomized trials may help elucidate issues such as the best chemotherapy regimen for second-line treatment.
\end{abstract}

\section{Contents}

1. Introduction

2. Second-line treatment after failed response to high-dose methotrexate

3. Conclusions

\section{Introduction}

Primary central nervous system lymphoma (PCNSL) is an extranodal form of non-Hodgkin's lymphoma arising in the craniospinal axis. For many years, PCNSL was reported to represent $3-5 \%$ of all primary central nervous system (CNS) tumors (1). However, the incidence of PCNSL appears to be increasing (2-4). PCNSL occurs at all ages, but is most common between 50 and 60 years of age, with a male to female ratio of

Correspondence to: Dr Ryuya Yamanaka, Research Center of Innovative Cancer Therapy, Kurume University School of Medicine, Asahimachi 67, Kurume, Fukuoka 830-0011, Japan

E-mail: ryaman@med.kurume-u.ac.jp

Key words: chemotherapy, primary central nervous system lymphoma, salvage therapy
1.5 (5). The symptoms are focal neurological deficits, mental disturbance and increased intracranial pressure. PCNSL can affect the brain, leptomeninges, spinal cord or eyes. Tumor manifestation is often diffuse and multifocal, most frequently affecting the supratentorial brain parenchyma, periventricular lesions involving the corpus callosum, basal ganglia, or thalamus. The characteristic radiographic finding of PCNSL is homogeneous contrast enhancement on MRI, at least $15 \mathrm{~mm}$ in contact with the subarachnoid space (6). Diffusion-weighted MRI (DW-MRI) and proton-MR-spectroscopy (1H-MRS) usually reveal a uniformly pathologic pattern of metabolite concentrations (6). Corticosteroids can temporarily induce regression of the tumor in $40-85 \%$ of patients (7). Diagnosis requires histologic confirmation, while molecular analysis of the rearrangement of immunoglobulin heavy chain genes by polymerase chain reaction and Southern blotting may also be acceptable (8). Most PCNSLs belong to the diffuse large B-cell and high grade Burkitt-like classifications, according to the Revised European-American Classification of Lymphoid Neoplasm (REAL) system (9).

Several Phase II studies combining chemotherapy with radiotherapy have been carried out in the last decade. The systemic use of high-dose methotrexate (HD-MTX)-based chemotherapy with radiation therapy for newly diagnosed PCNSL has improved the median overall survival from 25 to 51 months (10-15). Even without apparent leukoencephalopathy, the quality of life in surviving PCNSL patients is poor, and a proportion of patients suffer from mental deterioration following treatment (16). The reason for this is not clear, but the incidence appears to be higher in patients treated with radiotherapy than in those treated without it $(10,17)$. The risk of neurotoxicity is lower when chemotherapy alone is used to treat PCNSL (17). As a result, whole-brain radiation therapy (WBRT) is often deferred until tumor recurrence in patients who initially achieve complete remission through chemotherapy (18-21).

However, salvage treatment is necessary in $10-35 \%$ of patients with refractory disease and in at least another 30-60\% who suffer a relapse $(22,23)$. Furthermore, half of the 5-year survivors relapse 5-13 years after diagnosis (24). Moreover, the prognosis for patients who do not respond, or relapse after the initial response, is poor (23). Treatment options for patients with recurrent PCNSL are limited as there is no standard secondline chemotherapy. Salvage treatment, for which different strategies have been reported, may improve the outcome and 
quality of life, even if the optimal salvage regimen is unknown. Reni et al reported that median time to death was 14 months for patients who underwent salvage therapy and 2 months for untreated cases (23). New chemotherapeutic agents with clear activity in PCNSL are therefore needed for the treatment of recurrent disease. Twelve larger reports (25-36) with more than 10 patients were published between 2003 and 2008. This review analyzes the impact on survival of these reported second-line treatments for PCNSL, and provides some therapeutic recommendations for the management of patients with refractory or relapsed PCNSL.

\section{Second-line treatment after failed response to high-dose methotrexate}

\section{Monotherapy}

Radiation therapy. In patients who undergo chemotherapy alone, or for newly diagnosed PCNSL patients treated with low dose radiation therapy, radiation therapy may be selected for resistant or relapsed PCNSL. Nguyen et al reported the efficacy and toxicity of WBRT as salvage therapy for PCNSL in a retrospective study (25). The study included 27 patients (17 refractory and 10 recurrent) who failed with initial HD-MTX and then received salvage WBRT with a median dose of $36 \mathrm{~Gy}$. Ten patients (37\%) achieved a complete response (CR) and 10 patients (37\%) a partial response (PR) to WBRT, yielding a $74 \%$ overall response rate. Median survival from initiation of WBRT was 10.9 months (range 0.3-63.7 months) and median progression free survival (PFS) was 9.7 months. The univariate predictor of longer survival was an age of $<60$ years. Achievement of CR to WBRT within 4 months predicted longer survival. Late treatment-associated neurotoxicity was diagnosed in 4 patients $(15 \%)$ and was significantly associated with total radiation doses $>36 \mathrm{~Gy}$. No patient treated with daily fractions of $<1.8$ Gy developed late neurotoxicity. Hottinger et al also performed a retrospective study to evaluate the safety and efficacy of salvage WBRT (26). Forty-eight patients received salvage WBRT for PCNSL progression $(n=24)$ or recurrence $(n=24)$. After WBRT, 58\% achieved CR and 21\% achieved PR, yielding a $79 \%$ overall response rate. Median survival after WBRT was 16 months, 1 year survival after WBRT was 54\%, and median PFS was 10 months. An age of $<60$ years and CR to WBRT were associated with an improved outcome. Treatment-related neurotoxicity was observed in $22 \%$ of patients. Patients $>60$ years of age and those treatment-free for an interval of $<6$ months after MTX therapy were at increased risk for the development of neurotoxicity.

The median survival after salvage WBRT compares favorably with the 11.6 months reported for patients whose initial treatment was WBRT alone (37), suggesting that postWBRT survival is not diminished in MTX-resistant disease. However, in relapsed patients, the risk of neurotoxicity after salvage radiotherapy is potentially greater than with radiotherapy administered immediately after chemotherapy. This is because patients are older and have poor performance status at the relapse stage.

High-dose methotrexate. In a multicenter retrospective study of 22 patients who relapsed after initially achieving a CR to methotrexate, Plotkin et al reported that i) the median time to first relapse was 24.4 months (range 2-100 months); ii) reinduction with methotrexate at the same dose and schedule resulted in an overall response rate to first salvage of $91 \%$ (20/22 patients) and to second salvage of $100 \%$ (4/4 patients); iii) the median PFS was 61.9 months and the overall survival was 91.9 months in the selected group of patients (27). Toxicity was hematologic with 10 episodes of grade 3/4 during 566 cycles. However, HD-MTX should be avoided in patients who have received prior WBRT, or in patients with significant renal insufficiency. HD-MTX remains effective for relapsed PCNSL in patients who initially responded to HD-MTX. Given the low toxicity associated with HD-MTX, it is reasonable to consider deferring treatment with WBRT. According to the report, in patients treated with HD-MTX alone, neurotoxicity should also be observed.

Temozolomide. Temozolomide is a well-tolerated alkylating agent that is capable of permeating the blood-brain barrier (BBB) and has additive cytotoxicity when administered with radiotherapy. A Phase II trial assessing temozolomide $\left(150 \mathrm{mg} / \mathrm{m}^{2} /\right.$ day for 5 days every 28 days) in recurrent PCNSL patients previously treated with HD-MTX chemotherapy and/ or radiotherapy was evaluated (28). A median of 2 courses (range 1-12) of temozolomide was administered to 36 patients with a median age of 60 years, resulting in 9 CRs and 2 PRs, and a response rate of $31 \%$. One-year survival was $31 \%$, median PFS was 2.8 months and median survival time was 3.9 months. Although these results were not particularly encouraging, toxicity was negligible, with $9 \%$ CTC grade $3 / 4$ hematological toxicity. Temozolomide is active in recurrent PCNSL and should be evaluated further.

Rituximab. Rituximab, a chimeric monoclonal antibody against the CD20 antigen, has a molecular weight of $145 \mathrm{kDa}$ and achieves a cerebrospinal fluid (CSF) concentration of only $0.1 \%$ of matched serum levels after intravenous infusion (38). Rituximab improves survival when combined with CHOP therapy in patients with systemic diffuse large B-cell lymphoma (DLBCL) (39). Rubenstein et al conducted a Phase I study of intrathecal rituximab monotherapy (29). The protocol planned 9 injections of rituximab through an Ommaya reservoir in 10 patients. Meningeal responses were detected in 6 patients, 2 patients exhibited intraocular responses, and 1 of 6 cerebral lymphoma exhibited the resolution of parenchymal lymphoma, despite parenchymal progression in 4 patients. Intrathecal chemotherapy may not penetrate bulky leptomeningeal deposits and parenchymal lesions. The prominent contrast enhancement on neuroimaging that characterizes most PCNSLs is indicative of a disrupted $\mathrm{BBB}$, and significant endothelial abnormalities are observed pathologically in tumor vessels. Approximately $70-90 \%$ of PCNSL are DLBCL and its variant $(40,41)$, and rituximab is now part of combination therapy in some regimens for PCNSL. Systemic rituximab therapy in patients with relapsed or refractory PCNSL warrants further study combined with other chemotherapeutic agents.

Zevalin. ${ }^{90}$ Y-Ibritumomab tiuxetan (Zevalin) is a murine anti-CD20 covalently linked to the high-energy $\beta$-emitter, 
Yttrium-90, by the chelator tiuxetan. The efficacy of Zevalin to relapsed systemic non-Hodgkin's lymphoma has been demonstrated (42). Although monoclonal antibodies are large molecules excluded by an intact BBB, they may leak across permeable tumor vasculature into CNS lymphomas following radiolysis in both the targeted and adjacent lymphoma cells. Maza et al reported a Phase II trial to evaluate the therapeutic efficacy, toxicity and biodistribution of Zevalin in PCNSL (30). Ten patients with relapsed PCNSL were enrolled, 4 of whom responded. One patient had a CR lasting $\geq 30$ months, and 3 patients had a short-term response of $<4$ weeks. All patients experienced grade $3 / 4$ hematotoxicity, but no acute neurotoxicity. Penetration of a therapeutic antibody into PCNSL and significant clinical activity could be shown by single photon emission computed tomography imaging with ${ }^{111} \mathrm{In}$ Ibritumomab tiuxetan. Due to limited response duration and considerable hematotoxicity, future investigations should focus on a multimodal approach with additional chemotherapy.

Topotecan. Topotecan, a semi-synthetic derivative of camptothecin that can specifically inhibit topoisomerase I during the S-phase of the cell cycle, crosses the BBB to elicit documented efficacy in patients with relapsed systemic nonHodgkin's lymphoma (43). Topotecan penetrates the CNS, reaching concentrations in CSF of more than $30 \%$ in plasma (44). Fischer et al reported the results of a prospective study on topotecan chemotherapy in relapsed or refractory PCNSL (31). The study included 27 patients with a median age of 51 years. Fourteen patients were refractory and 13 relapsed after a median period of 6 months. Pre-treatment included chemotherapy in 26 patients and WBRT in 14. A 30-min daily topotecan infusion of $1.5 \mathrm{mg} / \mathrm{m}^{2}$ for 5 days was repeated every 3 weeks. The response rate was $33 \%$, with 5 CRs and 4 PRs. The median PFS was 2 months (9.1 months in responders), the overall survival was 8.4 months (9-28 months in responders), and 1 -year survival was $39 \%$. The survival times for resistant cases and recurrent cases were 9.5 and 3.4 months, respectively. Additional radiation therapy after topotecan treatment resulted in a longer survival time in the resistant patient group. However, with topotecan alone, 5 patients achieved long-term remission, lasting for 7.5, 10.5, 15.8, 24.1+ and 28.6 months, respectively. CTC grade 3/4 leucopenia or thrombocytopenia occurred in 26 and $11 \%$ of the patients, respectively. Eight of 12 surviving patients free of cerebral lymphoma for $>6$ months undergoing topotecan treatment exhibited signs of late neurotoxicity.

Voloschin et al reported a Phase II study that included 15 refractory or relapsed PCNSL patients with a median age of 56 years, treated with intravenous topotecan $(1.5 \mathrm{mg} /$ $\mathrm{m}^{2}$ ) for 5 consecutive days during each 21-day cycle (32). Three patients achieved CR and 3 achieved PR, yielding a total response rate of $40 \%$. The median PFS was 2 months. Eleven of the 15 patients had CTC grade $3 / 4$ neutropenia and 3 patients had grade 3 thrombocytopenia. Thirteen of the 15 patients received G-CSF during treatment. Topotecan as monotherapy is active in relapsed and refractory PCNSL with tolerable toxicity.

\section{Polychemotherapy}

Temozolomide + rituximab. Enting et al evaluated the efficacy of a combination of rituximab and temozolomide for recurrent or refractory PCNSL in a retrospective study (33). Treatment cycles over 28 days were: rituximab $\left(750 \mathrm{mg} / \mathrm{m}^{2}\right)$ on days 1 , 8,15 and 22 , and temozolomide $\left(100-200 \mathrm{mg} / \mathrm{m}^{2}\right)$ on days $1-7$ and 15-21. Fifteen patients with a median age of 69 years had a 53\% (6 CR, 2 PR) objective response rate with acceptable toxicity. Grade 3 thrombocytopenia was seen in 4 patients, grade 3 anemia in 1 and grade 3 leucopenia in 1 . No grade 4 toxicities were recorded. Median PFS for responding patients was 7.7 months. Median overall survival was 14 months, although median PFS for all patients was 2.2 months. This may reflect the third-line treatment after temozolomide and rituximab therapy. This combination merits further study and provides a reasonable therapeutic alternative for older patients with progressive PCNSL.

Carboplatin (intra-arterial) + cyclophosphamide + etoposide . Carboplatin, a second generation platinum analog, is an alkylating agent with efficacy in multiple cancers that has been used in combination with several agents for relapsed systemic lymphoma (45). The efficacy of cyclophosphamide and etoposide to newly diagnosed PCNSL has been shown $(41,46)$. The safety and efficacy of intra-arterial (IA) chemotherapy in conjunction with osmotic blood-brain-barrier disruption (BBBD) has been documented. The opening of tight junctions with mannitol allows increased levels of drugs (up to 100-fold) to reach the CNS (47-49). Tyson et al reported a retrospective study of patients with relapsed PCNSL treated with second line IA carboplatin-based chemotherapy plus BBBD (34). Thirtyseven relapsed PCNSL patients with a mean age of 57.5 years, most whom had failed with methotrexate-based chemotherapy, were enrolled. Mannitol was infused (5-10 ml/sec x $30 \mathrm{sec})$ into an internal carotid or vertebral artery. Cyclophosphamide was administered intravenously (i.v.) at a dose of 330-660 mg/m² 20 min before the mannitol infusion; subsequently, carboplatin was administered IA at a dose of $200-400 \mathrm{mg} / \mathrm{m}^{2}$ over $10 \mathrm{~min}$, starting within $5 \mathrm{~min}$ of the mannitol infusion. Etoposide was administered IA or i.v. at a dose of $200-400 \mathrm{mg} / \mathrm{m}^{2}$. This protocol was repeated every 4 weeks for up to 1 year. Nine patients had undergone prior radiotherapy. Nine patients had a radiographic $\mathrm{CR}$ and 4 patients had a PR, yielding an overall response rate of $35 \%$. The median time to failure for patients with CR and PR was 9.1 months. Overall median survival was 6.8 months (11-91 months). Six patients achieved survival times of 42.8, $50.4,50.9,54.7$ and 91 months from first carboplatin/BBBD treatment. Seven out of 37 patients survived longer than 27 months. However, a CTC grade 3/4 hematological adverse event was seen in $41 \%$ of the patients, and CTC grade $3 / 4$ non-hematological adverse events including ototoxicity (3\%), vascular toxicity $(11 \%)$, infection $(21 \%)$, pulmonary toxicity $(3 \%)$ and neurotoxicity $(11 \%)$ were noted.

Etoposide + ifosfamide + cytarabine (VIA). Ifosfamide, cytarabine (Ara-C) and etoposide (VP16) have been widely used for salvage therapy in systemic lymphomas $(50,51)$, and have been shown to be efficacious to newly diagnosed PCNSL $(41,46)$. At the doses described below as part of the VIA regimen, they are expected to reach cytotoxic levels in the $\mathrm{CSF}$ and brain parenchyma. Arellano-Rodrigo et al reported a retrospective study of 16 patients with a median age of 54 years comprising 1 refractory and 15 recurrent cases of PCNSL 
(35). All patients were initially treated homogeneously with a cyclophosphamide, doxorubicin, vincristine, dexamethasone/ carmustine, vincristine, cytarabine and methotrexate (CHOD/ BVAM) and radiotherapy regimen, followed by VP16, ifosfamide and Ara-C (VIA) chemotherapy as a salvage treatment. VIA included etoposide $\left(100 \mathrm{mg} / \mathrm{m}^{2}\right.$ on days $\left.1-3\right)$, ifosfamide (1000 mg/m² on days 1-5) and cytarabine (2000 mg/ $\mathrm{m}^{2} / 12 \mathrm{~h}$ on day 1$)$. The therapy was repeated every 28 days for a planned total of 6 cycles. Six patients (37\%) achieved CR. The median PFS was 5 months and 1-year overall survival was $41 \%$. The major toxicity was grade $2 / 4$ neutropenia in $69 \%$ and thrombocytopenia in $50 \%$ of patients. Five patients had grade $3 / 4$ infectious complications. One patient developed a reversible ifosfamide encephalopathy. The data presented indicate that VIA chemotherapy is an effective salvage regimen for patients with recurrent PCNSL.

\section{High-dose chemotherapy}

Intensive chemotherapy followed by autologous hematopoietic stem-cell rescue. Soussain et al reported the results of a prospective multicenter trial of intensive chemotherapy followed by autologous hematopoietic stem-cell rescue (IC + HCR) in patients with PCNSL following the failure of HD-MTX-based treatment (36). Salvage treatment consisted of 2 cycles of high-dose cytarabine and etoposide (CYVE). Intensive chemotherapy combined thiotepa, busulfan and cyclophosphamide. Forty-three patients with a median age of 52 years were included, with 22 relapse, 17 refractory and 4 partial response to first-line treatment cases. Twenty patients (47\%) were chemosensitive to CYVE; 15 of them proceeded to IC + HCR. IC + HCR was also administered to 12 patients who did not respond to CYVE. All but 1 of the 27 patients who underwent IC + HCR had complete remission. The median overall survival was 18.3 months in the overall population and 58.6 months among patients who completed IC + HCR. The respective median PFS times after IC + HCR were 11.6 and 41.1 months. Three patients died after CYVE as a result of septic shock $(n=2)$ or mesenteric necrosis $(n=1)$. Five patients had late neurologic toxicity after IC + HCR. Two of these patients had received first-line cranial radiotherapy before IC + HCR. The rationale for using CYVE and the combination of thiotepa-busulfan-cyclophosphamide for IC was that there was good CSF and brain diffusion of thiotepa and busulfan $(52,53)$. The CYVE regimen was used because of the lack of crossresistance between cytarabine, etoposide and methotrexate, and due to its efficacy in newly diagnosed PCNSL (54). IC $+\mathrm{HCR}$ is an effective treatment for refractory and recurrent PCNSL, and should be evaluated in comparative studies.

\section{New drugs}

Pemetrexed. In addition to inhibiting dihydrofolate reductase, pemetrexed targets other sites of inhibition, giving it a broader spectrum of activity than methotrexate (55). This suggests that pemetrexed may be effective in PCNSL. Altman et al reported a Phase II trial in 8 patients with recurrent PCNSL who were treated with pemetrexed $\left(900 \mathrm{mg} / \mathrm{m}^{2}\right)$ every 3 weeks (56). Most patients had large B-cell lymphoma, except for one who had a low-grade lymphoma. Most of the patients demonstrated a radiographic response, with the exception of the low-grade lymphoma patient, who had stable disease (SD).
The response rate was $62.5 \%$ (3 CR, 3 PR, 2 SD). The PFS of the CR cases was 3, 5 or 11 months. Five patients had CTC grade $3 / 4$ hematological toxicity and 3 patients CTC grade $3 / 4$ infection. This regimen exhibited minimal toxicity, however a potential neurotoxicity issue should be clarified since this drug is a dihydrofolate reductase inhibitor similar to methotrexate.

\section{Conclusions}

No standard therapy exists for relapsed PCNSL patients; however, numerous regimens have been used with variable results (Table I). Treatment options in such patients include WBRT; the reinduction of methotrexate in patients who previously responded to methotrexate; alternative chemotherapy agents including temozolomide, rituximab, procarbazine, nitrosourea, vincristine or cytarabine, or high dose chemotherapy followed by stem-cell rescue. A salvage treatment schedule should include drugs that can cross the BBB and agents with some efficacy as primary treatment, such as HD-MTX, cytarabine, vincristine, procarbazine or nitrosoureas (57-59). Radiotherapy constitutes an option for unirradiated patients. Re-treatment with HD-MTX may be an option in patients who relapse after prolonged initial CR to this agent and who did not receive prior radiotherapy. The use of temozolomide with or without rituximab in relapsed PCNSL after an initial chemoradiotherapy regimen is recommended in elderly patients. This may be performed with minimal toxicity. There are several papers supporting the effects of high-dose chemotherapy with autologous stem-cell transplantation, although these studies were for patients with PCNSL as first-line therapy $(60,61)$. Combination chemotherapy and high-dose chemotherapy with stem-cell transplantation should be encouraged in prospective trials with younger or better performance status patients, and as a salvage treatment in recurrent and refractory patients following a chemoradiotherapy regimen.

DLBCL is believed to be a heterogeneous diagnostic category (62). Gene-expression profiling studies of systemic lymphoma have distinguished 3 molecular subtypes of DLBCL known as 'germinal center B-like' (GCB) DLBCL, 'activated B-cell-like' (ABC) DLBCL and 'primary mediastinal B-cell lymphoma' (PMBL) (63-67). GCB DLBCLs seem to arise from normal germinal center B-cells, ABC DLBCLs may arise from postgerminal center B-cells that are arrested during plasmacytic differentiation, and PMBLs may arise from thymic B-cells (68). Patients with these DLBCL subtypes have significantly different survival rates following chemotherapy (63-66), leading to the current proposal that they represent distinct biological disease entities (68). The histogenetic derivation of PCNSL is poorly understood. Because the CNS lacks lymph nodes and lymphatics, it has been hypothesized that PCNSL may originate from B-cells derived from systemic lymphoid tissues that traffic in and out of the CNS (69). The molecular pathogenesis of PCNSL is also uncertain, but it is important that it is investigated further to enable the molecular classification and diagnosis of PCNSL. Ongoing studies should aim to clarify the clinical relevance of PCNSL phenotype heterogeneity in terms of the host's response to treatment and outcome, to identify biomarkers in a cohort of patients with PCNSL and to develop tailored therapy. 


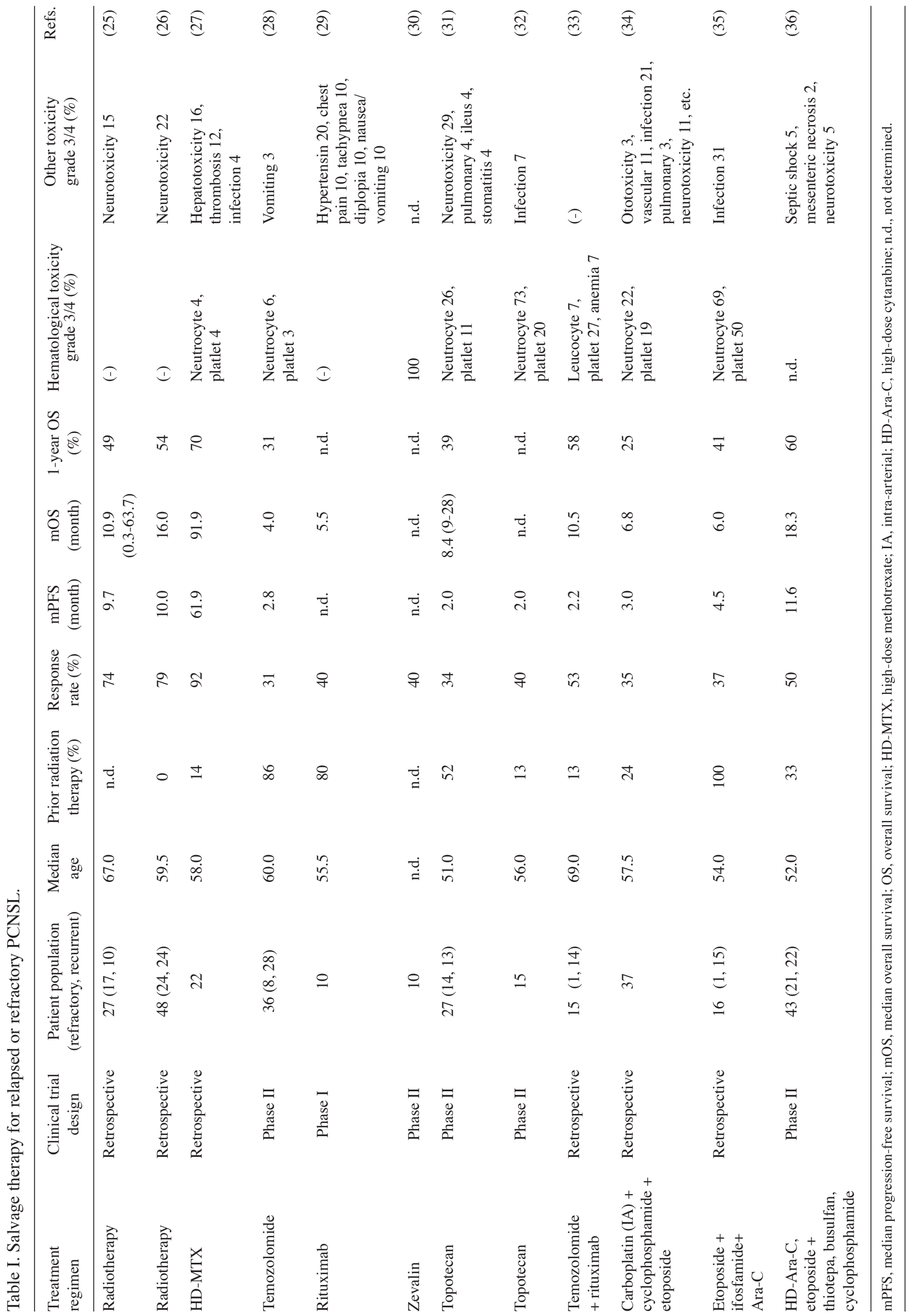


Apart from its rarity, there are several difficulties in treating PCNSL. For example, the aggressive course of relapsing PCNSL results in impaired performance status and neurological deterioration, usually in elderly patients. Because most reported studies have been retrospective, used undersized patient groups and involved heterogenous regimens, no conclusions can be drawn for an optimal regimen. Positive observations therefore remain to be confirmed in randomized trials. The inclusion of patients with relapsed or refractory PCNSL in prospective trials addressing new active agents or combinations is troublesome, but should be further recommended.

\section{References}

1. Panageas KS, Elkin EB, DeAngelis LM, Ben-Porat L and Abrey LE: Trends in survival from primary central nervous system lymphoma, 1975-1999: a population-based analysis. Cancer 104: 2466-2472, 2005.

2. Eby NL, Grufferman S, Flannelly CM, et al: Increasing incidence of primary brain lymphoma in the US. Cancer 62: 2461-2465, 1988.

3. Cote TR, Manns A, Hardy CR, Yellin FJ and Hartge P: Epidemiology of brain lymphoma among people with or without acquired immunodeficiency syndrome. AIDS/Cancer Study Group. J Natl Cancer Inst 88: 675-679, 1996.

4. Olson JE, Janney CA, Rao RD, et al: The continuing increase in the incidence of primary central nervous system non-Hodgkin's lymphoma: a surveillance, epidemiology and end results analysis. Cancer 95: 1504-1510, 2002.

5. Fine HA and Mayer RJ: Primary central nervous system lymphoma. Ann Intern Med 119: 1093-1104, 1993.

6. Küker W, Nägele T, Korfel A, et al: Primary central nervous system lymphomas (PCNSL): MRI features at presentation in 100 patients. J Neurooncol 72: 169-177, 2005.

7. Herrlinger U, Schabet M, Eichhorn M, et al: Prolonged corticosteroid-induced remission in primary central nervous system lymphoma: report of a case and review of the literature. Eur Neurol 36: 241-243, 1996

8. Endo S, Zhang SJ, Saito T, et al: Primary malignant lymphoma of the brain: mutation pattern of rearranged immunoglobulin heavy chain gene. Jpn J Cancer Res 93: 1308-1316, 2002.

9. Harris NL, Jaffe ES, Stein H, et al: A revised European-American classification of lymphoid neoplasms: a proposal from the International Lymphoma Study Group. Blood 84: 1361-1392, 1994.

10. Abrey LE, Yahalom J and DeAngelis LM: Treatment for primary CNS lymphoma: the next step. J Clin Oncol 18: 3144-3150, 2000.

11. DeAngelis LM, Yahalom J, Heinemann MH, et al: Primary CNS lymphoma: combined treatment with chemotherapy and radiotherapy. Neurology 40: 80-86, 1990.

12. Ferreri AJ, Reni M, Dell'Oro S, et al: Combined treatment with high-dose methotrexate, vincristine and procarbazine, without intrathecal chemotherapy, followed by consolidation radiotherapy for primary central nervous system lymphoma in immunocompetent patients. Oncology 60: 134-140, 2001.

13. Gavrilovic IT, Hormigo A, Yahalom J, DeAngelis LM and Abrey LE: Long-term follow-up of high-dose methotrexatebased therapy with and without whole brain irradiation for newly diagnosed primary CNS lymphoma. J Clin Oncol 24: 4570-4574, 2006.

14. Glass J, Gruber ML, Cher L and Hochberg FH: Preirradiation methotrexate chemotherapy of primary central nervous system lymphoma: long-term outcome. J Neurosurg 81: 188-195, 1994.

15. Poortmans PM, Kluin-Nelemans HC, Haaxma-Reiche H, et al: High-dose methotrexate-based chemotherapy followed by consolidating radiotherapy in non-AIDS-related primary centra nervous system lymphoma: European Organization for Research and Treatment of Cancer Lymphoma Group Phase II Trial 20962. J Clin Oncol 21: 4483-4488, 2003.

16. Abrey LE, DeAngelis LM and Yahalom J: Long-term survival in primary CNS lymphoma. J Clin Oncol 16: 859-863, 1998.

17. Freilich RJ, Delattre JY, Monjour A and DeAngelis LM: Chemotherapy without radiation therapy as initial treatment for primary CNS lymphoma in older patients. Neurology 46: 435-439, 1996.
18. Batchelor T, Carson K, O'Neill A, et al: Treatment of primary CNS lymphoma with methotrexate and deferred radiotherapy: a report of NABTT 96-07. J Clin Oncol 21: 1044-1049, 2003.

19. Cher L, Glass J, Harsh GR and Hochberg FH: Therapy of primary CNS lymphoma with methotrexate-based chemotherapy and deferred radiotherapy: preliminary results. Neurology 46: 1757-1759, 1996.

20. Guha-Thakurta N, Damek D, Pollack C, et al: Intravenous methotrexate as initial treatment for primary central nervous system lymphoma: response to therapy and quality of life of patients. J Neurooncol 43: 259-268, 1999.

21. McAllister LD, Doolittle ND, Guastadisegni PE, et al: Cognitive outcomes and long-term follow-up results after enhanced chemotherapy delivery for primary central nervous system lymphoma. Neurosurgery 46: 51-60, 2000.

22. Desablens B, Gardembas M and Haie-Meder CH: Primary central nervous system lymphoma: long term results of the GOELAMS LCP 88 trial with a focus on neurological complications among 152 patients. Ann Oncol 10: abs. 040, 1999.

23. Reni M, Ferreri AJ and Villa E: Second-line treatment for primary central nervous system lymphoma. Br J Cancer 79: 530-534, 1999.

24. Murray K, Kun L and Cox J: Primary malignant lymphoma of the central nervous system. Results of treatment of 11 cases and review of the literature. J Neurosurg 65: 600-607, 1986.

25. Nguyen PL, Chakravarti A, Finkelstein DM, et al: Results of whole-brain radiation as salvage of methotrexate failure for immunocompetent patients with primary CNS lymphoma. J Clin Oncol 23: 1507-1513, 2005.

26. Hottinger AF, DeAngelis LM, Yahalom J and Abrey LE: Salvage whole brain radiotherapy for recurrent or refractory primary CNS lymphoma. Neurology 69: 1178-1182, 2007.

27. Plotkin SR, Betensky RA, Hochberg FH, et al: Treatment of relapsed central nervous system lymphoma with high-dose methotrexate. Clin Cancer Res 10: 5643-5646, 2004.

28. Reni M, Zaja F, Mason W, et al: Temozolomide as salvage treatment in primary brain lymphomas. Br J Cancer 96: 864-867, 2007.

29. Rubenstein JL, Fridlyand J, Abrey L, et al: Phase I study of intraventricular administration of rituximab in patients with recurrent CNS and intraocular lymphoma. J Clin Oncol 25: 1350-1356, 2007.

30. Maza S, Kiewe P, Munz DL, et al: First report on a prospective trial with yttrium-90-labeled ibritumomab tiuxetan $\left(\right.$ Zevalin $\left.^{\mathrm{TM}}\right)$ in primary CNS lymphoma. Neuro Oncol 11: 423-429, 2009.

31. Fischer L, Thiel E, Klasen HA, et al: Prospective trial on topotecan salvage therapy in primary CNS lymphoma. Ann Oncol 17: 1141-1145, 2006.

32. Voloschin AD, Betensky R, Wen PY, Hochberg F and Batchelor T: Topotecan as salvage therapy for relapsed or refractory primary central nervous system lymphoma. J Neurooncol 86: 211-215, 2008.

33. Enting RH, Demopoulos A, DeAngelis LM and Abrey LE: Salvage therapy for primary CNS lymphoma with a combination of rituximab and temozolomide. Neurology 63: 901-903, 2004.

34. Tyson RM, Siegal T, Doolittle ND, et al: Current status and future of relapsed primary central nervous system lymphoma (PCNSL). Leuk Lymphoma 44: 627-633, 2003.

35. Arellano-Rodrigo E, López-Guillermo A, Bessell EM, et al: Salvage treatment with etoposide (VP-16), ifosfamide and cytarabine (Ara-C) for patients with recurrent primary central nervous system lymphoma. Eur J Haematol 70: 219-224, 2003.

36. Soussain C, Hoang-Xuan K, Taillandier L, et al: Intensive chemotherapy followed by hematopoietic stem-cell rescue for refractory and recurrent primary CNS and intraocular lymphoma. J Clin Oncol 26: 2512-2518, 2008.

37. Nelson DF, Martz KL, Bonner H, et al: Non-Hodgkin's lymphoma of the brain: can high dose, large volume radiation therapy improve survival? Report on a prospective trial by the Radiation Therapy Oncology Group (RTOG): RTOG 8315. Int J Radiat Oncol Biol Phys 23: 9-17, 1992.

38. Rubenstein JL, Combs D, Rosenberg J, et al: Rituximab therapy for CNS lymphomas: targeting the leptomeningeal compartment. Blood 101: 466-468, 2003.

39. Coiffier B, Lepage E, Briere J, et al: CHOP chemotherapy plus rituximab compared with $\mathrm{CHOP}$ alone in elderly patients with diffuse large-B-cell lymphoma. N Engl J Med 346: 235-242, 2002.

40. Shenkier TN, Voss N, Chhanabhai M, et al: The treatment of primary central nervous system lymphoma in 122 immunocompetent patients: a population-based study of successively treated cohorts from the British Colombia Cancer Agency. Cancer 103: 1008-1017, 2005. 
41. Yamanaka R, Morii K, Shinbo Y, et al: Results of treatment of 112 cases of primary CNS lymphoma. Jpn J Clin Oncol 38: 373-380, 2008.

42. Krishnan A, Nademanee A, Fung HC, et al: Phase II trial of a transplantation regimen of yttrium-90 ibritumomab tiuxetan and high-dose chemotherapy in patients with non-Hodgkin's lymphoma. J Clin Oncol 26: 90-95, 2008.

43. Kancherla RR, Nair JS, Ahmed T, et al: Evaluation of topotecan and etoposide for non-Hodgkin lymphoma: correlation of topoisomerase-DNA complex formation with clinical response. Cancer 91: 463-471, 2001.

44. Herben VM, Ten Bokkel Huinink WW and Beijnen JH: Clinical pharmacokinetics of topotecan. Clin Pharmacokinet 31: 85-102, 1996.

45. Zelenetz AD, Hamlin P, Kewalramani T, et al: Ifosfamide, carboplatin, etoposide (ICE)-based second-line chemotherapy for the management of relapsed and refractory aggressive non-Hodgkin's lymphoma. Ann Oncol 14: 5-10, 2003.

46. Pels H, Schmidt-Wolf IG, Glasmacher A, et al: Primary central nervous system lymphoma: results of a pilot and phase II study of systemic and intraventricular chemotherapy with deferred radiotherapy. J Clin Oncol 21: 4489-4495, 2003.

47. Kroll RA and Neuwelt EA: Outwitting the blood-brain barrier for therapeutic purposes: osmotic opening and other means. Neurosurgery 42: 1083-1099, 1998.

48. Neuwelt EA, Barnett PA, McCormick CI, et al: Differential permeability of a human brain tumor xenograft in the nude rat: impact of tumor size and method of administration on optimizing delivery of biologically diverse agents. Clin Cancer Res 4: 1549-1555, 1998.

49. Muldoon LL, Soussain C, Jahnke K, et al: Chemotherapy delivery issues in central nervous system malignancy: a reality check. J Clin Oncol 25: 2295-2305, 2007.

50. Cabanillas F, Hagemeister FB, Bodey GP and Freireich EJ: IMVP-16: an effective regimen for patients with lymphoma who have relapsed after initial combination chemotherapy. Blood 60: 693-697, 1982.

51. López R, Martino R, Brunet S, et al: Salvage chemotherapy with IAPVP-16 for advanced refractory or relapsed follicular lymphomas. Haematologica 84: 911-916, 1999.

52. Heideman RL, Cole DE, Balis F, et al: Phase I and pharmacokinetic evaluation of thiotepa in the cerebrospinal fluid and plasma of pediatric patients: evidence for dose-dependent plasma clearance of thiotepa. Cancer Res 49: 736-741, 1989

53. Hassan M, Oberg G, Ericson K, et al: In vivo distribution of [11C]-busulfan in cynomolgus monkey and in the brain of a human patient. Cancer Chemother Pharmacol 30: 81-85, 1992.

54. Soussain C, Patte C, Ostronoff M, et al: Small noncleaved cell lymphoma and leukemia in adults. A retrospective study of 65 adults treated with the LMB pediatric protocols. Blood 85: 664-674, 1995
55. Chattopadhyay S, Moran RG and Goldman ID: Pemetrexed: biochemical and cellular pharmacology, mechanisms and clinical applications. Mol Cancer Ther 6: 404-417, 2007.

56. Altman JK, Tellez C, Chandler J, et al: Phase II trial of recurrent primary CNS lymphoma (PCNSL) treated with pemetrexed. J Clin Oncol 26: abs. 2075, 2008.

57. DeAngelis LM, Yahalom J, Thaler HT and Kher U: Combined modality therapy for primary CNS lymphoma. J Clin Oncol 10: 635-643, 1992.

58. Bessell EM, Graus F, Punt JA, et al: Primary non-Hodgkin's lymphoma of the CNS treated with BVAM or CHOD/BVAM chemotherapy before radiotherapy. J Clin Oncol 14: 945-954, 1996.

59. Reni M, Ferreri AJ, Garancini MP and Villa E: Therapeutic management of primary central nervous system lymphoma in immunocompetent patients: results of a critical review of the literature. Ann Oncol 8: 227-234, 1997.

60. Illerhaus G, Marks R, Ihorst G, et al: High-dose chemotherapy with autologous stem-cell transplantation and hyperfractionated radiotherapy as first-line treatment of primary CNS lymphoma. J Clin Oncol 24: 3865-3870, 2006.

61. Montemurro M, Kiefer T, Schüler F, et al: Primary central nervous system lymphoma treated with high-dose methotrexate, high-dose busulfan/thiotepa, autologous stem-cell transplantation and response-adapted whole-brain radiotherapy: results of the multicenter Ostdeutsche Studiengruppe Hamato-Onkologie OSHO-53 phase II study. Ann Oncol 18: 665-671, 2007.

62. Coiffier B: Diffuse large cell lymphoma. Curr Opin Oncol 13 325-334, 2001.

63. Alizadeh AA, Eisen MB, Davis RE, et al: Distinct types of diffuse large B-cell lymphoma identified by gene expression profiling. Nature 403: 503-511, 2000.

64. Rosenwald A, Wright G, Chan WC, et al: The use of molecular profiling to predict survival after chemotherapy for diffuse large B-cell lymphoma. N Engl J Med 346: 1937-1947, 2002.

65. Wright G, Tan B, Rosenwald A, et al: A gene expression-based method to diagnose clinically distinct subgroups of diffuse large B-cell lymphoma. Proc Natl Acad Sci USA 100: 9991-9996, 2003.

66. Rosenwald A, Wright G, Leroy K, et al: Molecular diagnosis of primary mediastinal B cell lymphoma identifies a clinically favorable subgroup of diffuse large B-cell lymphoma related to Hodgkin lymphoma. J Exp Med 198: 851-862, 2003.

67. Savage KJ, Monti S, Kutok JL, et al: The molecular signature of mediastinal large B-cell lymphoma differs from that of other diffuse large B-cell lymphomas and shares features with classical Hodgkin lymphoma. Blood 102: 3871-3879, 2003.

68. Staudt LM and Dave S: The biology of human lymphoid malignancies revealed by gene expression profiling. Adv Immunol 87: 163-208, 2005.

69. Fine HA and Loeffler JS: Primary central nervous system lymphoma. In: The Lymphomas. Canellos GP, Lister TA and Sklar JL (eds). WB Saunders, Philadelphia, pp481-494, 1998. 